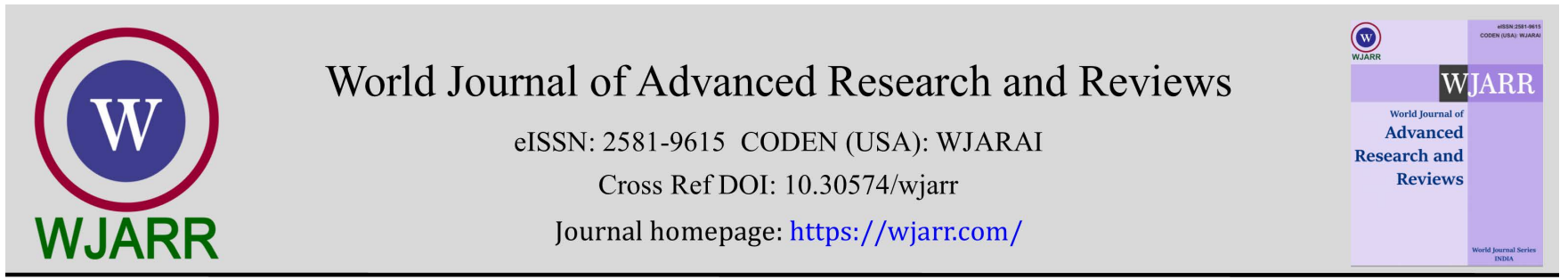

(RESEARCH ARTICLE)

Check for updates

\title{
Femoral neck bone autograft for the core decompression treatment of the hip osteonecrosis: Surgical technique
}

\author{
Panagiotis K. Karampinas ${ }^{1,}{ }^{*}$, Christos P. Zafeiris ${ }^{2}$ and John A. Vlamis ${ }^{3}$ \\ ${ }^{1}$ Hip and Knee Orthopaedic Department, Omilos Iatrikou, Psichico Clinic, Athens, Greece. \\ 2 Spine Orthopaedic Department, Metropolitan General, Athens, Greece. \\ $33^{\text {rd }}$ Orthopedic Department, University of Athens Medical School, KAT Athens Trauma Hospital, Greece.
}

World Journal of Advanced Research and Reviews, 2021, 10(02), 196-202

Publication history: Received on 07 April 2021; revised on 10 May 2021; accepted on 13 May 2021

Article DOI: https://doi.org/10.30574/wjarr.2021.10.2.0218

\begin{abstract}
Background: Hip joint preserving procedures delay the progression of the femoral head osteonecrosis. Nonvascularised autologous bone grafts have been used to fill the necrotic area and prevent the collapse of the femoral head. Harvesting and use of autologous femoral neck bone graft, minimizing the donor site complications and favoring the bone regeneration after core decompression.
\end{abstract}

Methods: 17 patients in early stage of the femoral head osteonecrosis were operated, 12 males and 5 females with mean age of 45.3 years old. Were treated with core decompression and autograft harvested from the femoral neck. All the patients were evaluated with an MRI preoperatively, and at the follow up with X-rays of the hip and functional clinical scores.

Results: We didn't observe any complications both at the donor site and the hip at any stage of the follow up. All the patients returned to previous everyday activities, free of pain. There was a significant improvement regarding the functional scores.

Conclusion: We present the surgical technique of using femoral neck bone autograft for the treatment of the femoral head osteonecrosis. The technique is simply and sufficient in most early and midterm cases adding an advantage to the minimal invasing and join preserving technique of core decompression.

Keywords: Osteonecrosis; Core decompression; Autograft; Femoral neck

\section{Introduction}

Osteonecrosis of the femoral head (ONFH) is one of the main reasons for total hip arthroplasty and revision procedures in young patients $[1,2]$. Hip joint preserving procedures are needed to delay the progression of the disease. The core decompression (CD) leads to a reduction in the intraosseous pressure and also stimulates stem cell regeneration. Nonvascularised bone grafts from different sources (allograft, autograft, or artificial) have been used to fill the necrotic area. The use of autologous bone graft prevents the collapse of the femoral head and the evolution of the ONFH $[3,4]$.

An ideal bone graft should provide osteoinductive and osteogenetic characteristics [5, 6]. The autologous bone graft satisfies all the requirements for good mode remodelling. Bone graft from femoral neck during the core decompression

\footnotetext{
${ }^{*}$ Corresponding author: Panagiotis Karampinas

Omilos Iatrikou, Athens Medical Center, Greece.

Copyright (C) 2021 Author(s) retain the copyright of this article. This article is published under the terms of the Creative Commons Attribution Liscense 4.0.
} 
provides good bone autograft regarding the quality of the graft and also the quantity of the autograft [4]. In addition, the donor site complications are eliminated.

The current paper aims to describe a surgical technique of harvesting and using autologous femoral neck bone graft, minimizing the donor site complications and favoring the bone regeneration after core decompression.

\section{Material and methods}

\subsection{Surgical Technique}

The procedure is performed in a supine position in the traction/trauma table and fluoroscopic guidance. A $1 \mathrm{~cm}$ lateral longitudinal skin incision was performed at the entrance point of the drill and controlled with a lateral and anteroposterior C-arm fluoroscopic view, targeting the necrotic area identified by the MRI (Fig1).

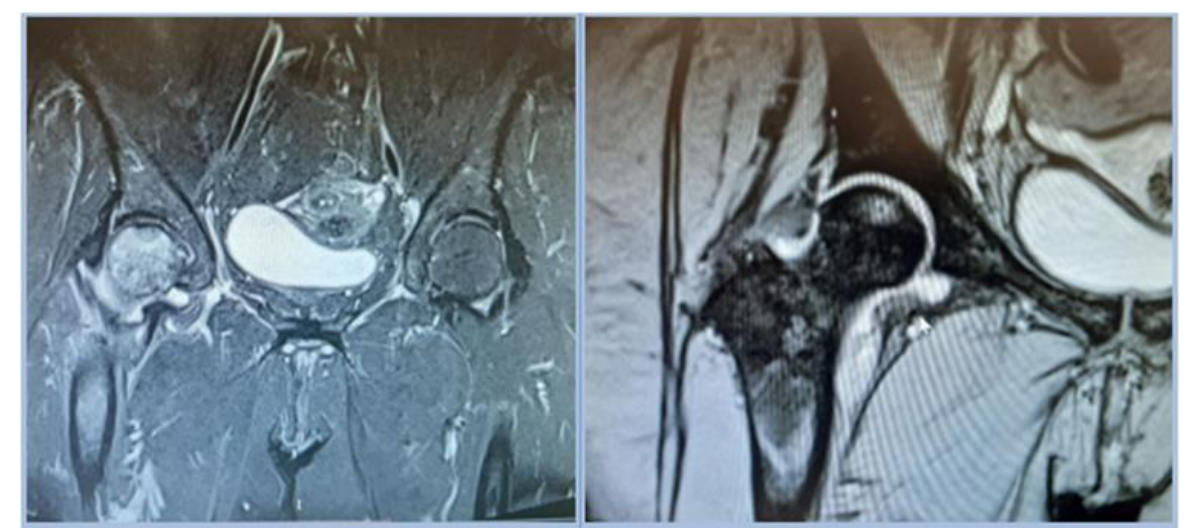

Figure 1 MRI of the right hip identifying the necrotic area in a patient with avascular necrosis (osteonecrosis) of the femoral head.

An incision of the subcutaneous tissue and the lateral fascia was performed, the vastus lateralis muscle was separated by blunt dissection, and the proximal lateral femoral cortex was exposed. A $3.2 \mathrm{~mm}$ guide wire is introduced at the entrance point and is guided forward the necrotic area, assisted by C-arm fluoroscopy. The 9 mm cannulated drill is introduced through the guide wire and used in contrast to previous techniques, to ream only the cortical part of the femur (Fig.2)

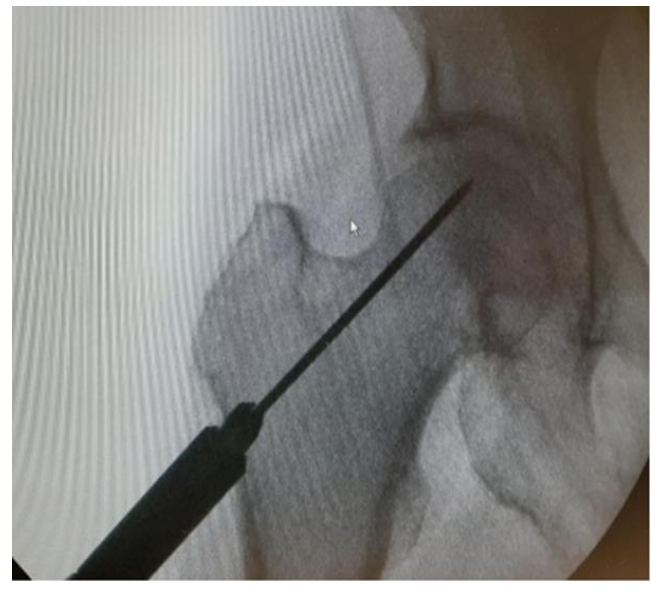

Figure 2 Femur lateral cortical entrance break point with a $9 \mathrm{~mm}$ cannulised drill.

At this point using a special trephine (ACL reconstruction Set of Zimmer-Biomet ${ }^{\mathrm{R}}$ ) is possible to harvest the cancellous bone from the femoral neck, introduced into the guide wire to prevent deviation from the pathway of the desired core's drill. The trephine is introduced and guided to the femoral neck arriving at the area before the necrotic zone of the femoral head by hammering. As a result, we collect a cylindrical cancellous bone with a length varying from 3.0 to 
$4.0 \mathrm{~cm}$ (length varying from $1 \mathrm{~cm}$ to $4 \mathrm{~cm}$ ). The diameter of the harvesting graft may vary according to the dimensions of the desired removing necrotic area during core decompression and calculated from the MRI study (Fig.3).

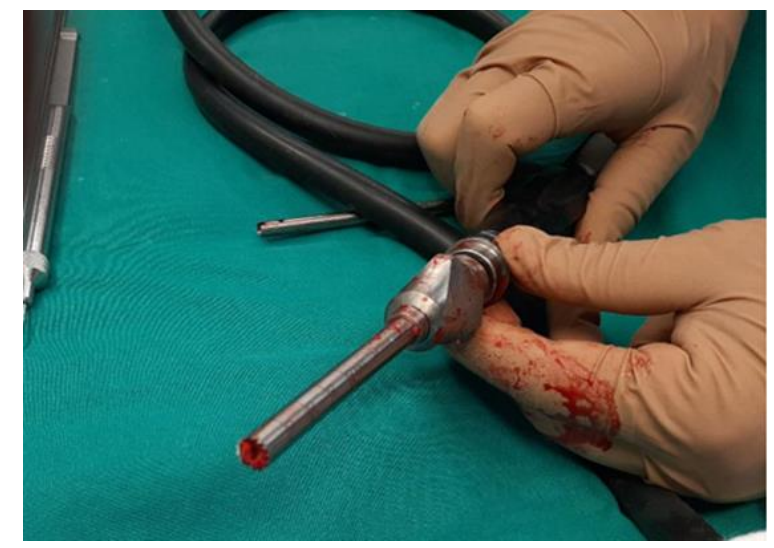

Figure 3 A special trephine (ACL Reconstruction Set of Zimmer-Biomet ${ }^{R}$ ) is possible to harvest the cancellous bone.

In the end, a final core drilling is performed with the initial $9.0 \mathrm{~mm}$ cannulated drill arriving at the center of the necrotic area and about 3 to $5 \mathrm{~mm}$ to the subchondral area (Fig.4).

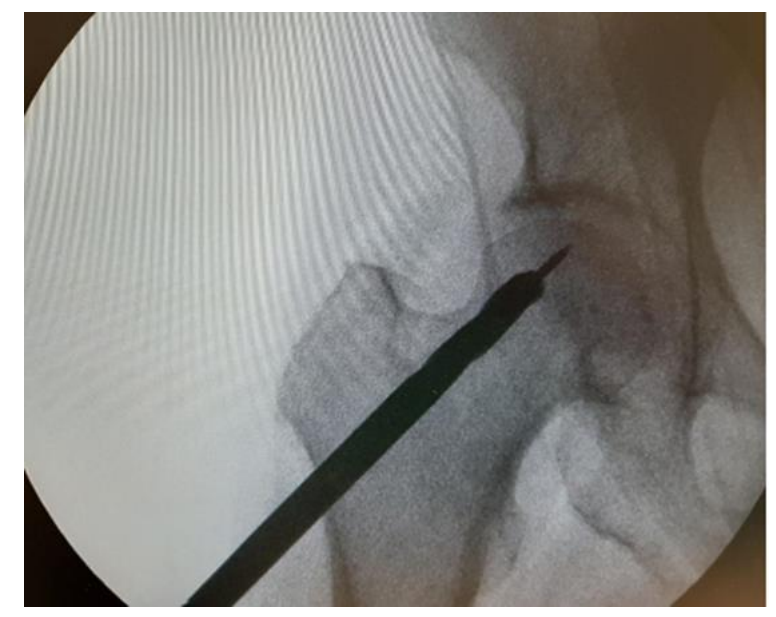

Figure 4 Femoral Neck and Head core drilling guided to the necrotic area of the femoral head.

The autologous bone graft is introduced, impacted into the reamed zone, and guided to the necrotic subchondral area (Fig 5).

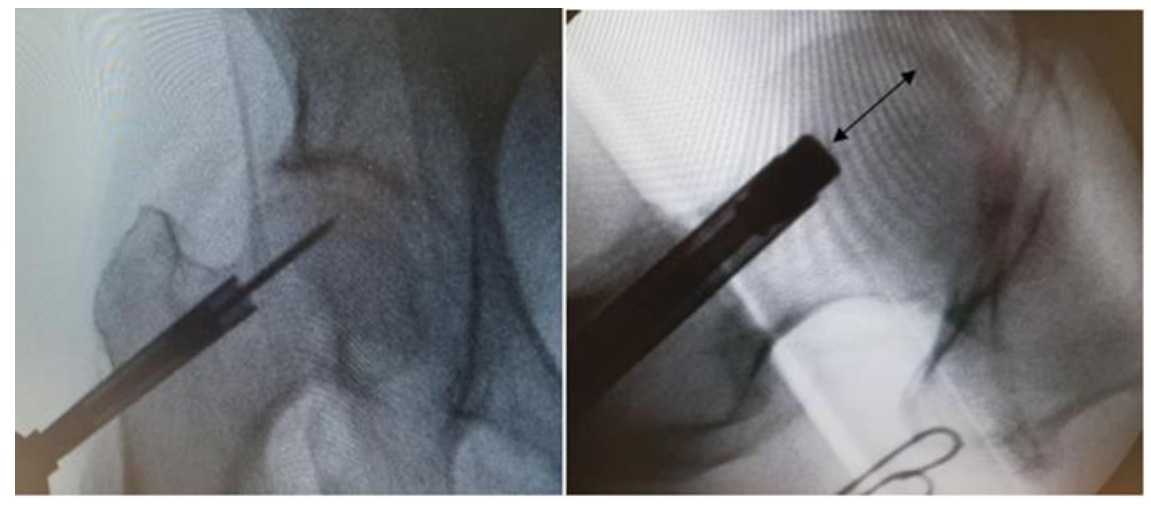

Figure 5 Introduction and impaction of the harvested bone graft to the necrotic subchondral area through the first guide wire. The arrow indicates the area occupied by the bone graft introduced. 
The necrotic area is critical to be removed completely by core decompression. Debridement of the core can be achieved by using a bigger size of a reamer or more aggressive and the corresponding size of bone graft harvest trephine (diameter varying from $7.0 \mathrm{~mm}$ to $10.0 \mathrm{~mm}$ ). The preoperative planning and the use of a proper reamer are necessary to achieve adequate removal of the necrotic area even if intraoperative endoscopic control of the core and magnetic resonance imaging (MRI) showed that removal of the necrotic tissue was rarely done [21, 22].

The patients rest in bed for $24 \mathrm{~h}$ and eventually mobilized with crunches and partial weight-bearing for a period of 4 weeks to favor the ingrowth. Antibiotic treatment was intravenously used within $24 \mathrm{~h}$ after surgery.

\section{Patients}

In our study were included 17 patients with ONFH from 2019 March until 2020 March. All the cases were operated by a senior orthopaedic surgeon. Preoperatively the patients were classified in early stage of ONFH (Ficat stage I [7] and II [10]), and were treated with core decompression and autografting of the necrotic area. There were 12 males and 5 females with mean age of 45.3 years old (range from 23 year to 54 year). In all cases the autograft was harvested by the femoral neck following the described technique. All the patients were studied preoperatively with radiological examination (anterolateral and lateral X-ray of the affected hip) and an MRI, to identify the necrotic area and the harvesting area of the femoral neck. The modified Harris Hip Score (m HHS) and pain visual analog scale (p VAS) were filled out. All the patients followed the same postoperative rehabilitation and pharmacological program. At the postoperative follow up all the patients were visited at 2 weeks, 8 weeks and 1 year after the treatment. They had a radiological control of the operated hip (anterolateral and lateral X-ray) and asked to fill out the functional questioners (m HHS and $\mathrm{p}$ VAS) at the 1 st year of the postoperative follow up.

\section{Results}

During a year follow up we didn't observe any complications both at the donor site (donor site infection and fracture, donor site morbidity) and the hip (collapse of the femoral articular surface), at any stage of the follow up. All the patients were able to return to everyday activities and they were free of pain. The harvesting technique of bone autograft from the femoral neck was successful with no complications both intraoperatively and postoperatively. There was a significant improvement regarding the functional scores (m HHS) from 74.5 preoperatively to 87.6 postoperatively. The pain VAS also was improved from 3.9 to 0.86 . The trephine allowed harvesting of a mean 7,2mm diameter bone cylinder (from $6 \mathrm{~mm}$ to $9 \mathrm{~mm}$ ). The mean length of the cylinder was $3.9 \mathrm{~cm}$ (from $3 \mathrm{~mm}$ to $5 \mathrm{~mm}$ ). The mean surgical time was 50 minutes.

\section{Discussion}

Core decompression (CD) is the most widely employed joint preserving technique to reduce pain and to prevent further deterioration. This technique was first described by Ficat and Arlet during their attempt to obtain biopsy specimens for confirmation of the diagnosis in the pre-MRI era [7]. Common joint preserving surgical procedures for the treatment of ONFH include core decompression, core decompression with autologous bone marrow (CD+BM), and core decompression with autologous bone grafting $(\mathrm{CD}+\mathrm{AB})[8]$. There were also reported techniques as core decompression with vascular pedicle bone graft, core decompression combined with bone debridement, and core decompression combined with tantalum rod implantation, all of which achieved satisfactory clinical results [9]. Core's decompression common complications are heterotopic ossifications (33.3\%), pain (21.7\%), and fracture (18.8\%), with an overall rate of $4.79 \%$. The rate of radiographic progression of the two methods ( $C D+B M$ and $C D+A B)$ compared to $C D$ defined the success rate ( $C D+B M$ success rate $74.0 \%, C D+A B$ success rate $81.0 \%)$. In the same study, compare of the success rates of the included Ficat I, II, and III cases, concluded that the rate was Ficat I> II > III, and the difference was statistically significant. The success rate of stage III is only $27.44 \%$ and for that reason, the CD should be considered carefully for cases of stage III [8].

Core decompression aims to reduce the intraosseous pressure, remove the necrotic tissue and stimulate the formation of blood vessels around the decompression tunnel, enhance the replacement of the new bone, and delay the progression of osteonecrosis [10]. A study confirms that CD combined with cytotherapy obtains a relatively efficient treatment for reducing the failure rate of early and mid-term ONFH patients [11]. Another study confirmed that CD combined with autologous bone marrow stem cells has achieved good results in early ONFH patients [12]. It has also been shown that the levels of osteoprogenitor cells in the hematopoietic and stromal marrow compartments are decreased in this disease $[13,14]$. A decrease in the mesenchymal stem-cell pool of the proximal aspect of the femur might not provide enough osteoblasts to meet the needs of bone-remodelling in the early stage of the disease [15]. The lack of sufficient 
osteoprogenitor cells compromises the ability of the bone to heal and remodel. In the very early stages of osteonecrosis, providing sufficient repair capacity through the implantation of osteogenic cells could make these lesions reversible $[15,16]$.

Spontaneous regression of avascular necrosis is rare, with the vast majority of untreated patients progressing to THA and a collapse rate of $67 \%$ in asymptomatic patients and $85 \%$ of symptomatic hips [17]. In clinical practice, preventing the collapse of subchondral bone has to be given sufficient biomechanical support and suitable circumstances to fulfil bone repair. The bone graft placed at the suffering subchondral area of the femoral head supports the area avoiding the femoral's head collapse and evolve to hip joint arthritis [19]. To enhance osseous regeneration, the application of osteogenic or angiogenic precursor cells (or both) in combination with or without supporting growth factors remains an appealing possibility. Adult tissue-derived, multipotent mesenchymal stem cells (MSCs) represent an exceptionally promising candidate for cell-based therapeutic approaches [27]. The quality of presented stem cells determines whether the defective self-repair in the necrotic area can be corrected to enhance repair [23]. Many studies demonstrate that CD in combination with the application of autologous bone marrow aspirate concentrates into the femoral head seems to be a safe and efficient treatment alternative in the early stages of AVN of the femoral head when compared to CD alone and lower the THA conversion rate [24, 25, 26, 27, 28 and 29]. The presence of vital bone favors the vascularisation, facilitating the growth of the blood vessel along the tunnel into the femoral head and promoting the repair of the femoral head and the formation of new bone. A variety of growth factors produced by osteogenic cells, platelets, and inflammatory cells - including Bone Morphogenetic Proteins (BMPs), insulin-like growth factor-1 and -2, transforming growth factor- $\beta 1$ (TGF- $\beta 1$ ), platelet-derived growth factor, and fibroblast growth factor-2-are functionally involved in bone healing. The bone matrix serves as a reservoir for these growth factors, which are activated during matrix resorption by matrix metalloproteases. Additionally, the acidic environment that develops during the inflammatory process leads to activation of latent growth factors, which assist in chemo-attraction, migration, proliferation, and differentiation of MSCs into osteoblasts or chondroblasts [27]. The autologous bone graft satisfies all the requirements with osteoinductive and osteogenetic characteristics for good mode remodelling $[5,6]$.

The goal of this procedure is to provide mechanical support of the articular surface while promoting bone healing and remodelling and delay or avoid total hip replacement. The use of joint preserving techniques to delay the evolution of the disease and minimally invasive techniques to harvest bone graft is a necessity. Autologous bone graft from the femoral neck eliminates the prolonged operation time, the increased trauma area of the patient, the insufficient bone supply, and many complications in the donor site (iliac crest morbidity). Larger defects, therefore, continue to prove difficult to treat. The initial as well as the remaining necrotic volume of the femoral head influence the outcome [18, 20]. In these cases, the use of combining an allograft remains a one-way choice.

\section{Conclusion}

The use of autologous femoral neck bone graft increase the success rate providing an optimal bone stimulator, avoiding the subchondral fracture, and eliminating the donor site complications observed in cases of iliac crest autograft harvesting well known in the literature. The use of a trephine through a guide wire makes the femoral neck graft harvesting simply and sufficient in most early and midterm cases adding an advantage to the minimal invasing and join preserving technique of core decompression.

\section{Compliance with ethical standards}

Acknowledgments

None

\section{Disclosure of conflict of interest}

The authors declare no conflict of interest.

\section{Statement of informed consent}

Informed consent was obtained from all individual participants included in the study. 


\section{References}

[1] Adelani MA, Keeney JA, Palisch A, Fowler SA, Clohisy JC. Has total hip arthroplasty in patients 30 years or younger improved? A systematic review. Clin Orthop Relat Res. Aug 2013; 471(8): 2595-601.

[2] Kurtz S, Ong K, Lau E, Mowat F, Halpern M. Projections of primary and revision hip and knee arthroplasty in the United States from 2005 to 2030. J Bone Joint Surg Am. Apr 2007; 89(4): 780-5.

[3] Xu S, Zhang L, Jin H, Shan L, Zhou L, Xiao L, Tong P. Autologous stem cells combined core decompression for treatment of avascular necrosis of the femoral head: a systematic meta-analysis. BioMed research international. 2017 Aug 3; 2017.

[4] Landgraeber S, Warwas S, Claßen T, Jäger M. Modifications to advanced Core decompression for treatment of Avascular necrosis of the femoral head. BMC Musculoskelet Disord. 21 Nov 2017; 18(1): 479.

[5] Costantino PD, Friedman CD. Synthetic bone graft substitutes. Otolaryngol Clin North Am. Oct 1994; 27(5): 103774.

[6] TJ Cypher, JP Grossman. Biological principles of bone graft healing. J Foot Ankle Surg. Sep-0ct 1996; 35(5): 4137.

[7] Ficat Pr. Idiopathic bone necrosis of the femoral head. Early diagnosis and treatment. J Bone Joint Surg Br. Jan 1985; 67(1): 3-9.

[8] Kun-chi Hua, Xiong-gang Yang, Jiang-tao Feng, Feng Wang, Li Yang, Hao Zhang and Yong-cheng Hu. The efficacy and safety of core decompression for the treatment of femoral head necrosis: a systematic review and metaanalysis. J Orthop Surg Res. 11 Sep 2019; 14(1): 306.

[9] David R Marker, Thorsten M Seyler, Slif D Ulrich, Siddharth Srivastava, Michael A Mont. Do modern techniques improve core decompression outcomes for hip osteonecrosis? Clin Orthop Relat Res. May 2008; 466(5): 1093103.

[10] ME Steinberg, PG Larcom, B Strafford, WB Hosick, A Corces, RE Bands, KE Hartman. Core decompression with bone grafting for osteonecrosis of the femoral head. Clin Orthop Relat Res. May 2001; (386): 71-8.

[11] Xiao Yu, Difeng Zhang, Xianjun Chen, Ji Yang, Lin Shi, Qingjiang Pang . Effectiveness of various hip preservation treatments for non-traumatic osteonecrosis of the femoral head: a network meta-analysis of randomized controlled trials. J Orthop Sci. Mar 2018; 23(2): 356-364.

[12] Xu S, Zhang L, Jin H, Shan L, Zhou L, Xiao L, Tong P. Autologous stem cells combined core decompression for treatment of avascular necrosis of the femoral head: a systematic meta-analysis. Biomed Res Int. 2017; 1-11.

[13] Gangji V, de maertelaer V, hauzeur J-P. Autologous bone marrow cell implantation in the treatment of nontraumatic osteonecrosis of the femoral head: five year follow-up of a prospective controlled study. Bone. Nov 2011; 49(5): 1005-9.

[14] Gangji V, toungouz m, hauzeur J-P. Stem cell therapy for osteonecrosis of the femoral head. Expert Opin Biol Ther. Apr 2005; 5(4): 437-42.

[15] Inoue A, Ono K. A histological study of idiopathic avascular necrosis of the head of the femur. J Bone Joint Surg Br. May 1979; 61-B (2): 138-43.

[16] Hernigou P, Beaujean F, Lambotte JC. Decrease in the mesenchymal stemcell pool in the proximal femur in corticosteroid-induced osteonecrosis. J Bone Joint Surg Br. Mar 1999; 81(2): 349-55.

[17] Hauzeur JP, Pasteels JL. Pathology of bone marrow distant from the sequestrum in nontraumatic aseptic necrosis of the femoral head. Bone circulation and bone necrosis. Berlin: Springer. 1990; 73-6.

[18] Rijnen WH, Gardeniers JW, Buma P, Yamano K, Slooff TJ, Schreurs BW. Treatment of femoral head osteonecrosis using bone impaction grafting. Clin Orthop Relat Res. Dec 2003; (417): 74-83.

[19] Hungerford DS. Bone marrow pressure, venography and core decompression in ischemic necrosis of the femoral head. The Hip: Proceedings of the Seventh Open Scientific Meeting of The Hip Society. St Louis, MO: CV Mosby; 1979: 218-237.

[20] SB Keizer, NB Kock, PDS Dijkstra, AHM Taminiau, RGH H Nelissen. Treatment of avascular necrosis of the hip by a non-vascularised cortical graft. J Bone Joint Surg Br. Apr 2006; 88(4): 460-6. 
[21] Tim Classen, Sebastian Warwas, Marcus Jäger and Stefan Landgraeber. Two-year follow-up after advanced core decompression. J Tissue Eng Regen Med. Apr 2017; 11(4): 1308-1314.

[22] Landgraeber S, Theysohn JM, Classen T, Jäger M, Warwas S, Hohn HP, Kowalczyk W. Advanced core decompression, new treatment option of avascular necrosis of the femoral head - a first follow-up. J Tissue Eng Regen Med. Nov 2013; 7(11): 893-900.

[23] John Vlamis, Panagiotis Karampinas, Eustratios Kavroudakis, Spiros Pneumaticos. The use of core track endoscopy to document accurate decompression of the femoral head. Hip International. May-Jun 2014; 24(3): 284-289.

[24] Zhan Yu Wu1, Qi Sun, Ming Liu, Brian E. Grottkau, Zhi Xu He, Qiang Zou and Chuan Ye. Correlation between the efficacy of stem cell therapy for osteonecrosis of the femoral head and cell viability. BMC Musculoskelet Disord. 29 Jan 2020; 21(1): 55.

[25] Hakan Pilge,Bernd Bittersohl, Johannes Sch. Bone marrow aspirate concentrate in combination with intravenous iloprost increases bone healing in patients with avascular necrosis of the femoral head: a matched pair analysis. Orthop Rev (Pavia). 4 Jan 2017; 8(4): 6902.

[26] Joon Soon Kang1, Young Ju Suh2, Kyoung Ho Moon, Jun Sung Park, Tae Hoon Roh, Myung Hoon Park and Dong Jin Ryu. Clinical efficiency of bone marrow mesenchymal stem cell implantation for osteonecrosis of the femoral head: a matched pair control study with simple core decompression. Stem Cell Res Ther. 25 Oct 2018; 9(1): 274.

[27] Matthew T Houdek, Cody C wyles, John R Martin, Rafael J Sierra. Stem cell treatment for avascular necrosis of the femoral head: current perspectives. Stem Cells Cloning. 9 Apr 2014; 7: 65-70.

[28] Lars Rackwitz, Lars Eden, Stephan Reppenhagen, Johannes C Reichert, Franz Jakob, Heike Walles, Oliver Pullig, Rocky S Tuan, Maximilian Rudert and Ulrich Nöth. Stem cell- and growth factor-based regenerative therapies for avascular necrosis of the femoral head. Stem Cell Res Ther. 22 Feb 2012; 3(1): 7.

[29] Luca Andriolo, Giulia Merli, Carlos Tobar, Sante Alessandro Altamura, Elizaveta Kon, Giuseppe Filardo. Regenerative therapies increase survivorship of avascular necrosis of the femoral head: a systematic review and meta-analysis. Int Orthop. Jul 2018; 42(7): 1689-1704. 